BOW-PH-151

BRX-TH-639

\title{
One-loop SYM-supergravity relation for five-point amplitudes
}

\author{
Stephen G. Naculich ${ }^{1}, a$ and Howard J. Schnitzer $2 . b$ \\ ${ }^{a}$ Department of Physics \\ Bowdoin College, Brunswick, ME 04011, USA \\ ${ }^{b}$ Theoretical Physics Group \\ Martin Fisher School of Physics \\ Brandeis University, Waltham, MA 02454, USA \\ naculich@bowdoin.edu, schnitzr@brandeis.edu
}

\begin{abstract}
We derive a linear relation between the one-loop five-point amplitude of $\mathcal{N}=8$ supergravity and the one-loop five-point subleading-color amplitudes of $\mathcal{N}=4$ supersymmetric Yang-Mills theory.
\end{abstract}

\footnotetext{
${ }^{1}$ Research supported in part by the NSF under grant PHY-0756518

${ }^{2}$ Research supported in part by the DOE under grant DE-FG02-92ER40706
} 


\section{Introduction}

The recent explosion of interest in $\mathcal{N}=4$ supersymmetric Yang-Mills (SYM) and $\mathcal{N}=8$ supergravity amplitudes is due to the discovery of a host of symmetries and structures that are completely hidden in the traditional Feynman diagram approach to computing amplitudes. Perhaps even more surprising are the relations that have begun to emerge between perturbative gauge theory and gravity amplitudes.

Tree-level relations between gauge and graviton amplitudes were originally discovered in the field-theory limit of the string-theoretic KLT relations [1]. One-loop relations between $\mathcal{N}=4$ $\mathrm{SYM}$ and $\mathcal{N}=8$ supergravity amplitudes were also first obtained using string theory [2], while unitarity methods have been used to derive relations at higher loops [3 6]. While much of the recent spectacular progress has involved planar (large- $N$ ) SYM amplitudes, the connection between SYM and supergravity amplitudes intimately involves the non-planar (subleading-in$1 / N)$ contributions to SYM amplitudes.

The connection between gauge and gravity amplitudes has recently been significantly tightened through the discovery of a new color-kinematic duality of gauge theory amplitudes by Bern, Carrasco, and Johansson [7,8]. It was conjectured [7] and proven [9] that tree-level $n$-gluon amplitudes may be written as a sum over diagrams built from cubic vertices

$$
\mathcal{A}=\sum_{i} \frac{n_{i} c_{i}}{d_{i}}
$$

in which the kinematic numerators $n_{i}$ obey precisely the same set of algebraic relations observed by the color factors $c_{i}$. The denominators $d_{i}$ are products of the inverse propagators corresponding to each cubic diagram. Given a tree-level $n$-gluon amplitude in a form that respects colorkinematic duality, it was further conjectured [7] and proven [13] that $n$-graviton amplitudes can be expressed as a sum over the same diagrams

$$
\mathcal{M}=\sum_{i} \frac{\tilde{n}_{i} n_{i}}{d_{i}}
$$

with the color factors replaced by a second copy $\tilde{n}_{i}$ of the numerator factors. At the loop level, color-kinematic duality was conjectured [7,8] to hold for the numerator factors appearing in the integrands of gauge-theory loop diagrams built from cubic vertices, and this was verified through three loops for the $\mathcal{N}=4 \mathrm{SYM}$ four-point amplitude [8, 14]. Loop-level gravity amplitudes can then be obtained from the same diagrams using a double copy of the numerator factors. Related work on color-kinematic duality appears in refs. [15, 16], and some recent reviews are refs. [14,17].

Recently, Carrasco and Johansson [18] demonstrated that the five-point amplitude can be written in a form in which the numerators of the integrands respect color-kinematic duality at one, two [18], and three [19] loops. The one-loop $\mathcal{N}=4$ SYM five-point amplitude is [18]

$$
\mathcal{A}_{5}^{(1)}=i g^{5} \sum_{S_{5}}\left(\frac{1}{10} \beta_{12345} I^{(P)}(12345) C_{12345}^{(P)}+\frac{1}{4} \gamma_{12} I^{(B)}(12 ; 345) C_{12 ; 345}^{(B)}\right)
$$


where $I^{(P)}$ and $I^{(B)}$ are the scalar pentagon and box integrals defined below in eqs. (3.5) and (3.6), $C^{(P)}$ and $C^{(B)}$ are the corresponding color factors defined in eqs. (2.1) and (3.2), and 3

$$
\beta_{12345}=\delta^{(8)}(Q) \frac{[12][23][34][45][51]}{4 \varepsilon(1,2,3,4)}, \quad \quad \gamma_{12}=\delta^{(8)}(Q) \frac{[12]^{2}[34][45][35]}{4 \varepsilon(1,2,3,4)}
$$

where $[i j]$ are the usual helicity spinor products, $\varepsilon(1,2,3,4) \equiv \varepsilon_{\mu \nu \rho \sigma} k_{1}^{\mu} k_{2}^{\nu} k_{3}^{\rho} k_{4}^{\sigma}$, and $\delta^{(8)}(Q)$ is the manifestly-supersymmetric delta function of the Grassmann-valued supermomentum. The BCJ conjecture [8] then suggests that the corresponding one-loop $\mathcal{N}=8$ supergravity five-point amplitude is [18]

$$
\mathcal{M}_{5}^{(1)}=-\left(\frac{\kappa}{2}\right)^{5} \sum_{S_{5}}\left(\frac{1}{10} \tilde{\beta}_{12345} \beta_{12345} I^{(P)}(12345)+\frac{1}{4} \tilde{\gamma}_{12} \gamma_{12} I^{(B)}(12 ; 345)\right)
$$

where $\tilde{\beta}_{12345}$ and $\tilde{\gamma}_{12}$ are numerator factors of a second copy of $\mathcal{N}=4 \mathrm{SYM}$, and it was verified [18,20] that eq. (1.5) agrees with the known expression in ref. [4].

Loop amplitudes of $\mathcal{N}=4 \mathrm{SYM}$ theory possess IR divergences which can be regulated through dimensional regularization in $D=4-2 \epsilon$ dimensions with $\epsilon<0$. The leading IR divergence of one-loop $n$-point amplitudes, such as eq. (1.3), goes as $1 / \epsilon^{2}[21]$. In a $1 / N$ expansion of the SYM amplitude, however, the coefficients of the subleading-color amplitudes have IR divergences that are less severe than the leading-color (planar) amplitudes [22 24]. At one loop, for example, the IR divergence of the double-trace amplitude (which is subleading in $1 / N$ ) goes as $1 / \epsilon$, as ascertained for $n$-point functions in ref. 24]. This matches the leading IR divergence of one-loop $\mathcal{N}=8$ supergravity amplitudes, which also go as $1 / \epsilon$ [25], suggesting the possibility of linear relations between supergravity amplitudes and subleading-color SYM amplitudes. Such relations were found at one and two loops for four-point amplitudes in ref. [22]. In this paper, we propose and prove the relation

$$
\mathcal{M}_{5}^{(1)}=\frac{-1}{20 i g^{5}}\left(\frac{\kappa}{2}\right)^{5} \sum_{S_{5}} \tilde{\beta}_{12345} A_{5 ; 3}(12 ; 345)
$$

between the one-loop $\mathcal{N}=8$ supergravity five-point amplitude $\mathcal{M}_{5}^{(1)}$ and the subleading-color SYM amplitude $A_{5 ; 3}(12 ; 345)$, defined as the coefficient of the double-trace term in the trace expansion of the one-loop $\mathcal{N}=4 \mathrm{SYM}$ five-point amplitude $\mathcal{A}_{5}^{(1)}$ (cf. eq. (2.5)). This relation is distinct from one proposed in ref. [20] between the one-loop supergravity amplitude and the leading-color SYM amplitudes $A_{5 ; 1}(12345)$, as we discuss at the end of sec. 4 .

The relation between loop-level gauge theory and supergravity amplitudes proposed in ref. [8] is a diagram-by-diagram map between the integrands. In contrast, the various relations between subleading-color SYM and supergravity amplitudes found for one- and two-loop four-point amplitudes in ref. [22], for one-loop four- and five-point amplitudes in ref. [20], and for one-loop five-point amplitudes in the present paper, are between the integrated amplitudes. We observe that in all of these cases, the kinematic numerators are independent of the loop momenta and

\footnotetext{
${ }^{3} \mathrm{~A}$ representation of the tree-level five-point amplitude in terms of $\beta_{12345}$ and $\gamma_{12}$ was presented in ref. [16].
} 


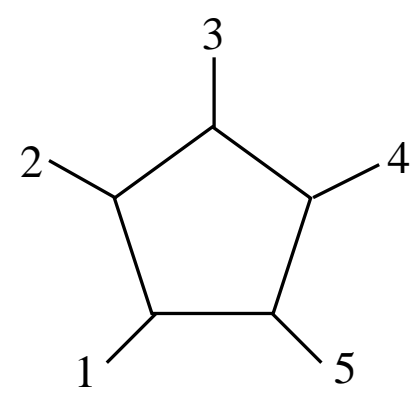

(a)

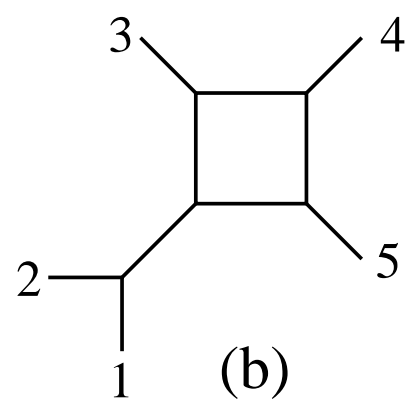

Figure 1: One-loop five-point diagrams

therefore can be factored out, leaving the same set of scalar integrals contributing to both the SYM and supergravity amplitudes. Finding relations between the integrated SYM and supergravity amplitudes when the numerator factors are dependent on the loop momenta will be more challenging.

This paper is organized as follows. In section 2, we review the color and trace bases for representing SYM amplitudes. In section 3, we decompose the Carrasco-Johannson representation of the one-loop five-point amplitude into the trace basis. In section 4, we present and prove a linear relation between the one-loop five-point supergravity amplitude and the one-loop subleadingcolor five-point SYM amplitudes. Section 5 contains our conclusions, and in an appendix we review the leading IR divergence of the one-loop subleading-color five-point SYM amplitude.

\section{The one-loop $\mathcal{N}=4$ SYM five-point amplitude}

In this section, we review how the one-loop five-point amplitude of $\mathcal{N}=4$ SYM theory may be expressed in both the color basis and the trace basis, and how the coefficients in each of these bases are related to one another.

A complete color basis for one-loop five-point amplitudes is spanned by the pentagon color diagram in fig. 1(a)

$$
C_{12345}^{(P)}=\tilde{f}^{g a_{1} b} \tilde{f}^{b a_{2} c} \tilde{f}^{c a_{3} d} \tilde{f}^{d a_{4} e} \tilde{f}^{e a_{5} g}
$$

and its permutations (e.g., see ref. [26]), where $\tilde{f}^{a b c}=i \sqrt{2} f^{a b c}$ are the structure constants for the $\mathrm{SU}(N)$ gauge group. The one-loop five-point amplitude can then be expressed as

$$
\mathcal{A}_{5}^{(1)}=\sum_{S_{5}} \frac{1}{10} a(12345) C_{12345}^{(P)}
$$

where $a(12345)$ depends on the momenta and helicities of the external states, and the sum is over all permutations of the momentum, helicity, and color index $a$ of the external states. The color factor $C_{12345}^{(P)}$ is manifestly symmetric under cyclic permutations of 12345 and antisymmetric under $12345 \rightarrow 54321$. Bose symmetry implies that $a(12345)$ is therefore also symmetric under 
cyclic permutations and antisymmetric under reversal of indices, so we can rewrite the amplitude as a sum over twelve terms

$$
\mathcal{A}_{5}^{(1)}=\sum_{S_{5} / \mathbb{Z}_{5} \times \mathbb{Z}_{2}} a(12345) C_{12345}^{(P)}=\sum_{i=1}^{12} a_{i} c_{i}
$$

where we choose an explicit basis

$$
c_{i}=\left\{C_{12345}^{(P)}, C_{14325}^{(P)}, C_{13425}^{(P)}, C_{12435}^{(P)}, C_{14235}^{(P)}, C_{13245}^{(P)}, C_{12543}^{(P)}, C_{14523}^{(P)}, C_{13524}^{(P)}, C_{12534}^{(P)}, C_{14532}^{(P)}, C_{13542}^{(P)}\right\}
$$

for later convenience.

Alternatively, the one-loop five-point amplitude may be expressed in terms of a trace basis [27]

$$
\begin{aligned}
\mathcal{A}_{5}^{(1)}= & \sum_{S_{5} / \mathbb{Z}_{5} \times \mathbb{Z}_{2}} A_{5 ; 1}(12345) N[\operatorname{Tr}(12345)-\operatorname{Tr}(54321)] \\
& +\sum_{S_{5} / \mathbb{Z}_{2} \times S_{3}} A_{5 ; 3}(12 ; 345) \operatorname{Tr}(12)[\operatorname{Tr}(345)-\operatorname{Tr}(543)]
\end{aligned}
$$

where $\operatorname{Tr}(12345) \equiv \operatorname{Tr}\left(T^{a_{1}} T^{a_{2}} T^{a_{3}} T^{a_{4}} T^{a_{5}}\right)$ and the matrices $T^{a}$ are the generators in the defining representation of $\mathrm{SU}(N)$, normalized according to $\operatorname{Tr}\left(T^{a} T^{b}\right)=\delta^{a b}$.

There are twelve independent single-trace coefficients $A_{5 ; 1}(12345)$, which are symmetric under cyclic permutations and antisymmetric under reversal of indices, and ten independent doubletrace coefficients $A_{5 ; 3}(12 ; 345)$, which are symmetric under exchange of the first two indices, and completely antisymmetric under permutations of the last three indices. We therefore write

$$
\mathcal{A}_{5}^{(1)}=\sum_{\lambda=1}^{22} A_{\lambda} t_{\lambda}
$$

where the basis $\left\{t_{\lambda}\right\}$ consists of the following single-trace terms

$$
\begin{array}{ll}
t_{1}=N[\operatorname{Tr}(12345)-\operatorname{Tr}(15432)] & t_{7}=N[\operatorname{Tr}(12543)-\operatorname{Tr}(13452)] \\
t_{2}=N[\operatorname{Tr}(14325)-\operatorname{Tr}(15234)] & t_{8}=N[\operatorname{Tr}(14523)-\operatorname{Tr}(13254)] \\
t_{3}=N[\operatorname{Tr}(13425)-\operatorname{Tr}(15243)] & t_{9}=N[\operatorname{Tr}(13524)-\operatorname{Tr}(14253)] \\
t_{4}=N[\operatorname{Tr}(12435)-\operatorname{Tr}(15342)] & t_{10}=N[\operatorname{Tr}(12534)-\operatorname{Tr}(14352)] \\
t_{5}=N[\operatorname{Tr}(14235)-\operatorname{Tr}(15324)] & t_{11}=N[\operatorname{Tr}(14532)-\operatorname{Tr}(12354)] \\
t_{6}=N[\operatorname{Tr}(13245)-\operatorname{Tr}(15423)] & t_{12}=N[\operatorname{Tr}(13542)-\operatorname{Tr}(12453)]
\end{array}
$$

and the following double-trace terms

$$
\begin{array}{ll}
t_{13}=\operatorname{Tr}(12)[\operatorname{Tr}(345)-\operatorname{Tr}(543)] & t_{18}=\operatorname{Tr}(13)[\operatorname{Tr}(245)-\operatorname{Tr}(542)] \\
t_{14}=\operatorname{Tr}(23)[\operatorname{Tr}(451)-\operatorname{Tr}(154)] & t_{19}=\operatorname{Tr}(24)[\operatorname{Tr}(351)-\operatorname{Tr}(153)] \\
t_{15}=\operatorname{Tr}(34)[\operatorname{Tr}(512)-\operatorname{Tr}(215)] & t_{20}=\operatorname{Tr}(35)[\operatorname{Tr}(412)-\operatorname{Tr}(214)] \\
t_{16}=\operatorname{Tr}(45)[\operatorname{Tr}(123)-\operatorname{Tr}(321)] & t_{21}=\operatorname{Tr}(41)[\operatorname{Tr}(523)-\operatorname{Tr}(325)] \\
t_{17}=\operatorname{Tr}(51)[\operatorname{Tr}(234)-\operatorname{Tr}(432)] & t_{22}=\operatorname{Tr}(52)[\operatorname{Tr}(134)-\operatorname{Tr}(431)] .
\end{array}
$$


The color (2.4) and trace bases (2.7) and (2.8) are related by

$$
c_{i}=\sum_{\lambda=1}^{22} M_{i \lambda} t_{\lambda} .
$$

Using $\tilde{f}^{a b c}=\operatorname{Tr}\left(\left[T^{a}, T^{b}\right] T^{c}\right)$ to evaluate eq. (2.1) yields

$$
c_{1}=t_{1}+\sum_{\lambda=13}^{22} t_{\lambda}
$$

which gives the first row $M_{1 \lambda}$ of the transformation matrix. The other rows may be obtained through permutations of the external state indices

$$
M_{i \lambda}=\left(\begin{array}{rrrrrrrrrrrrrrrrrrrrrr}
1 & 0 & 0 & 0 & 0 & 0 & 0 & 0 & 0 & 0 & 0 & 0 & 1 & 1 & 1 & 1 & 1 & 1 & 1 & 1 & 1 & 1 \\
0 & 1 & 0 & 0 & 0 & 0 & 0 & 0 & 0 & 0 & 0 & 0 & -1 & 1 & 1 & -1 & -1 & -1 & 1 & -1 & -1 & -1 \\
0 & 0 & 1 & 0 & 0 & 0 & 0 & 0 & 0 & 0 & 0 & 0 & 1 & 1 & 1 & -1 & 1 & -1 & 1 & -1 & -1 & 1 \\
0 & 0 & 0 & 1 & 0 & 0 & 0 & 0 & 0 & 0 & 0 & 0 & -1 & 1 & 1 & 1 & -1 & 1 & 1 & 1 & 1 & -1 \\
0 & 0 & 0 & 0 & 1 & 0 & 0 & 0 & 0 & 0 & 0 & 0 & -1 & 1 & 1 & 1 & 1 & -1 & 1 & -1 & 1 & -1 \\
0 & 0 & 0 & 0 & 0 & 1 & 0 & 0 & 0 & 0 & 0 & 0 & 1 & 1 & 1 & -1 & -1 & 1 & 1 & 1 & -1 & 1 \\
0 & 0 & 0 & 0 & 0 & 0 & 1 & 0 & 0 & 0 & 0 & 0 & -1 & -1 & 1 & 1 & -1 & -1 & -1 & 1 & -1 & -1 \\
0 & 0 & 0 & 0 & 0 & 0 & 0 & 1 & 0 & 0 & 0 & 0 & 1 & 1 & -1 & 1 & 1 & 1 & -1 & -1 & 1 & -1 \\
0 & 0 & 0 & 0 & 0 & 0 & 0 & 0 & 1 & 0 & 0 & 0 & -1 & -1 & -1 & -1 & -1 & 1 & 1 & 1 & 1 & 1 \\
0 & 0 & 0 & 0 & 0 & 0 & 0 & 0 & 0 & 1 & 0 & 0 & 1 & -1 & 1 & 1 & 1 & -1 & -1 & 1 & -1 & 1 \\
0 & 0 & 0 & 0 & 0 & 0 & 0 & 0 & 0 & 0 & 1 & 0 & 1 & 1 & -1 & -1 & -1 & 1 & -1 & -1 & -1 & -1 \\
0 & 0 & 0 & 0 & 0 & 0 & 0 & 0 & 0 & 0 & 0 & 1 & -1 & -1 & -1 & -1 & 1 & -1 & 1 & -1 & 1 & 1
\end{array}\right)
$$

Equation (2.9) implies that the coefficients of the color and trace bases are related by

$$
A_{\lambda}=\sum_{i=1}^{12} a_{i} M_{i \lambda}
$$

hence

$$
A_{i}=a_{i}, \quad i=1, \cdots, 12
$$

i.e., the coefficients $a(12345)$ in the color basis (2.3) are precisely equal to $A_{5 ; 1}(12345)$, the coefficients of the single-trace terms in eq. (2.5) . On the other hand, these planar amplitudes are well-known to be given by the sum of five $1 \mathrm{~m}$ scalar box integrals [21]. The leading IR divergence of these planar amplitudes therefore goes as $1 / \epsilon^{2}$.

Equations (2.11) and (2.12) also give the coefficients of the double-trace terms; e.g.,

$$
A_{13}=a_{1}-a_{2}+a_{3}-a_{4}-a_{5}+a_{6}-a_{7}+a_{8}-a_{9}+a_{10}+a_{11}-a_{12} .
$$

Comparing eqs. (2.13) and (2.14) we see that each of the double-trace amplitudes can be written as a linear combination of the single-trace amplitudes, a result long known [21,27]. An equivalent 
way to derive this result is to observe that the rank-twelve matrix (2.11) possesses ten null eigenvectors $R_{\lambda j}$ :

$$
\sum_{\lambda=1}^{22} M_{i \lambda} R_{\lambda j}=0, \quad j=1, \cdots 10 .
$$

Given that $M_{i \lambda}$ has the form $\left(\begin{array}{ll}\mathbb{1}_{12 \times 12} & m\end{array}\right)$, one sees that $R=\left(\begin{array}{c}-m \\ \mathbb{1}_{10 \times 10}\end{array}\right)$. The existence of these null eigenvectors, together with eq. (2.12), implies ten relations among the color-ordered amplitudes

$$
\sum_{\lambda=1}^{22} A_{\lambda} R_{\lambda j}=0, \quad j=1, \cdots 10 .
$$

For example, the $j=1$ relation is

$$
A_{13}=A_{1}-A_{2}+A_{3}-A_{4}-A_{5}+A_{6}-A_{7}+A_{8}-A_{9}+A_{10}+A_{11}-A_{12}
$$

that is,

$$
\begin{aligned}
A_{5 ; 3}(12 ; 345)= & +A_{5 ; 1}(12345)-A_{5 ; 1}(14325)+A_{5 ; 1}(13425)-A_{5 ; 1}(12435) \\
& -A_{5 ; 1}(14235)+A_{5 ; 1}(13245)-A_{5 ; 1}(12543)+A_{5 ; 1}(14523) \\
& -A_{5 ; 1}(13524)+A_{5 ; 1}(12534)+A_{5 ; 1}(14532)-A_{5 ; 1}(13542) .
\end{aligned}
$$

Using the symmetries of the single-trace amplitudes, this can be written

$$
\begin{aligned}
A_{5 ; 3}(12 ; 345)= & +A_{5 ; 1}(12345)+A_{5 ; 1}(23415)+A_{5 ; 1}(13425)+A_{5 ; 1}(34215) \\
& +A_{5 ; 1}(32415)+A_{5 ; 1}(13245)+A_{5 ; 1}(21345)+A_{5 ; 1}(23145) \\
& +A_{5 ; 1}(31425)+A_{5 ; 1}(34125)+A_{5 ; 1}(32145)+A_{5 ; 1}(31245)
\end{aligned}
$$

precisely the set of cyclically-ordered permutations in eq. (7.3) of ref. [21].

Although the planar amplitudes $A_{5 ; 1}(12345)$ have a leading $1 / \epsilon^{2}$ IR divergence, eq. (2.18) implies that the leading IR divergence of the subleading-color amplitude $A_{5 ; 3}(12 ; 345)$ is only $1 / \epsilon$, as shown in ref. [24]. We rederive this result in an appendix of this paper.

\section{The CJ representation of the five-point amplitude}

Carrasco and Johansson recently derived expressions for one- and two-loop $\mathcal{N}=4$ SYM fivepoint amplitudes that manifest color-kinematic duality [18]. At one-loop their ansatz takes the form 8

$$
\mathcal{A}_{5}^{(1)}=i g^{5} \sum_{i} \int \frac{d^{D} p}{(2 \pi)^{D}} \frac{1}{S_{i}} \frac{N_{i} C_{i}}{\prod_{m} l_{i_{m}}^{2}}
$$

where the sum is over all cubic one-loop five-point diagrams, including relabelings of the external lines, with symmetry factors $S_{i}$. The $l_{i_{m}}$ are the momenta flowing through each of the internal 
legs of the diagram, which can depend on the external momenta $k_{j}$ and the loop momentum $p$. The numerator factors $N_{i}$ are as-yet-unspecified functions of momenta and helicity. The color factors $C_{i}$ include the pentagon color diagram previously specified in eq. (2.1), but to obtain a representation of the amplitude that satisfies color-kinematic duality, one must also include the box-plus-line color diagram in fig. 1)(b)

$$
C_{12 ; 345}^{(B)}=\tilde{f}^{a_{1} a_{2} b} \tilde{f}^{b c g} \tilde{f}^{c a_{3} d} \tilde{f}^{d a_{4} e} \tilde{f}^{e a_{5} g}
$$

which is manifestly antisymmetric under $1 \leftrightarrow 2$ and symmetric under $3 \leftrightarrow 5$. The box color diagrams are not independent of the pentagon color diagrams; the Jacobi identity implies

$$
C_{12 ; 345}^{(B)}=C_{12345}^{(P)}+C_{12543}^{(P)} \text {. }
$$

Other color diagrams containing triangles and bubbles need not be included since the corresponding numerator factors $N_{i}$ vanish for $\mathcal{N}=4$ SYM theory.

In a beautiful analysis employing supersymmmetry, generalized unitarity, and color-kinematic duality of the numerator factors $N^{(P)}$ and $N^{(B)}$, Carrasco and Johannson showed that the numerator factors at one loop are independent of the loop momentum, 4 and so eq. (3.1) can be written as

$$
\mathcal{A}_{5}^{(1)}=i g^{5} \sum_{S_{5}}\left(\frac{1}{10} \beta_{12345} I^{(P)}(12345) C_{12345}^{(P)}+\frac{1}{4} \gamma_{12 ; 345} I^{(B)}(12 ; 345) C_{12 ; 345}^{(B)}\right)
$$

where $I^{(P)}$ and $I^{(B)}$ are the scalar integrals shown in fig. 1

$$
\begin{aligned}
I^{(\mathrm{P})}(12345) & =\int \frac{d^{D} p}{(2 \pi)^{D}} \frac{1}{p^{2}\left(p+k_{1}\right)^{2}\left(p+k_{1}+k_{2}\right)^{2}\left(p-k_{4}-k_{5}\right)^{2}\left(p-k_{5}\right)^{2}}, \\
I^{(\mathrm{B})}(12345) & =\frac{1}{\left(k_{1}+k_{2}\right)^{2}} \int \frac{d^{D} p}{(2 \pi)^{D}} \frac{1}{p^{2}\left(p+k_{1}+k_{2}\right)^{2}\left(p-k_{4}-k_{5}\right)^{2}\left(p-k_{5}\right)^{2}},
\end{aligned}
$$

and $\beta_{12345}$ and $\gamma_{12 ; 345}$ are functions of external momenta and helicities given by eq. (1.4), although we will not need their explicit forms in what follows.

One may see from eqs. (3.5) and $(3.6)$ that $I^{(P)}(12345)$ is invariant under cyclic permutations and reversals of 12345 , and $I^{(B)}(12 ; 345)$ is invariant under $1 \leftrightarrow 2$ and $3 \leftrightarrow 5$. Hence $5 \beta_{12345}$ is symmetric under cyclic permutations and antisymmetric under reversal of indices, and $\gamma_{12 ; 345}$ is antisymmetric under $1 \leftrightarrow 2$ and symmetric under $3 \leftrightarrow 5$. On the basis of these symmetries, we rewrite eq. (3.4) as

$$
\mathcal{A}_{5}^{(1)}=i g^{5}\left(\sum_{S_{5} / \mathbb{Z}_{5} \times \mathbb{Z}_{2}} \beta_{12345} I^{(P)}(12345) C_{12345}^{(P)}+\sum_{S_{5} / \mathbb{Z}_{2} \times \mathbb{Z}_{2}} \gamma_{12 ; 345} I^{(B)}(12 ; 345) C_{12 ; 345}^{(B)}\right) .
$$

\footnotetext{
${ }^{4}$ For the two-loop five-point amplitude, the numerator factors do include dependence on the loop momenta.

${ }^{5}$ As we will see below, $\beta_{12345}$ and $\gamma_{12 ; 345}$ possess further symmetries as well.
} 
We now recast the Carrasco-Johansson one-loop amplitude into the trace basis. First we substitute eq. (3.3) into eq. (3.4) to obtain

$$
\begin{aligned}
\mathcal{A}_{5}^{(1)}= & i g^{5} \sum_{S_{5}}\left(\frac{1}{10} \beta_{12345} I^{(P)}(12345)+\frac{1}{2} \gamma_{12 ; 345} I^{(B)}(12 ; 345)\right) C_{12345}^{(P)} \\
= & i g^{5} \sum_{S_{5} / \mathbb{Z}_{5} \times \mathbb{Z}_{2}}\left(\beta_{12345} I^{(P)}(12345)+\left[\gamma_{12 ; 345} I^{(B)}(12 ; 345)+\gamma_{23 ; 451} I^{(B)}(23 ; 451)+\right.\right. \\
& \left.\left.\quad+\gamma_{34 ; 512} I^{(B)}(34 ; 512)+\gamma_{45 ; 123} I^{(B)}(45 ; 123)+\gamma_{51 ; 234} I^{(B)}(51 ; 234)\right]\right) C_{12345}^{(P)} .
\end{aligned}
$$

By comparing with eqs. (2.3) and (2.13), one sees that the one-loop planar amplitude is given by

$$
\begin{aligned}
A_{5 ; 1}(12345)=i g^{5} & \left(\beta_{12345} I^{(P)}(12345)+\left[\gamma_{12 ; 345} I^{(B)}(12 ; 345)+\gamma_{23 ; 451} I^{(B)}(23 ; 451)+\right.\right. \\
& \left.\left.+\gamma_{34 ; 512} I^{(B)}(34 ; 512)+\gamma_{45 ; 123} I^{(B)}(45 ; 123)+\gamma_{51 ; 234} I^{(B)}(51 ; 234)\right]\right) .
\end{aligned}
$$

The double-trace color-ordered amplitude follows from eq. (2.18):

$$
\begin{aligned}
A_{5 ; 3}(12 ; 345)=i g^{5}( & +\beta_{12345} I^{(P)}(12345)-\beta_{14325} I^{(P)}(14325)+\beta_{13425} I^{(P)}(13425) \\
& -\beta_{12435} I^{(P)}(12435)-\beta_{14235} I^{(P)}(14235)+\beta_{13245} I^{(P)}(13245) \\
& -\beta_{12543} I^{(P)}(12543)+\beta_{14523} I^{(P)}(14523)-\beta_{13524} I^{(P)}(13524) \\
& +\beta_{12534} I^{(P)}(12534)+\beta_{14532} I^{(P)}(14532)-\beta_{13542} I^{(P)}(13542) \\
& +2\left[\gamma_{34 ; 512} I^{(B)}(34 ; 512)+\gamma_{34 ; 125} I^{(B)}(34 ; 125)+\gamma_{34 ; 251} I^{(B)}(34 ; 251)\right] \\
& +2\left[\gamma_{45 ; 312} I^{(B)}(45 ; 312)+\gamma_{45 ; 123} I^{(B)}(45 ; 123)+\gamma_{45 ; 231} I^{(B)}(45 ; 231)\right] \\
& \left.+2\left[\gamma_{53 ; 412} I^{(B)}(53 ; 412)+\gamma_{53 ; 124} I^{(B)}(53 ; 124)+\gamma_{53 ; 241} I^{(B)}(53 ; 241)\right]\right) .
\end{aligned}
$$

Carrasco and Johansson derived a number of additional relations satisfied by the numerator functions $\beta_{12345}$ and $\gamma_{12: 345}$. In addition to the symmetries noted above, $\gamma_{12 ; 345}$ is invariant under $3 \leftrightarrow 4$, and therefore under all permutations of 345 , and so can be denoted simply as $\gamma_{12}=-\gamma_{21}$. The box integral contribution to the double-trace amplitude (3.10) can therefore be expressed as

$$
\left.A_{5 ; 3}(12 ; 345)\right|_{\mathrm{box}}=2 i g^{5}\left[\gamma_{34} I^{(B)}(34)+\gamma_{45} I^{(B)}(45)+\gamma_{53} I^{(B)}(53)\right]
$$

where $I^{(B)}(12)$ represents the sum of box integrals

$$
I^{(B)}(12) \equiv I^{(B)}(12 ; 345)+I^{(B)}(12 ; 453)+I^{(B)}(12 ; 534)
$$

which is invariant under $1 \leftrightarrow 2$ and all permutations of 345 . 
In addition to the above symmetres, the $\gamma_{i j}$ obey

$$
\sum_{j=1}^{5} \gamma_{i j}=0
$$

so that there are only six linearly-independent functions: $\gamma_{12}, \gamma_{13}, \gamma_{14}, \gamma_{23}, \gamma_{24}$, and $\gamma_{34}$. The $\beta_{12345}$ are expressible in terms of the $\gamma_{i j}$ :

$$
\beta_{12345}=\frac{1}{2}\left(\gamma_{12}+\gamma_{13}+\gamma_{14}+\gamma_{23}+\gamma_{24}+\gamma_{34}\right)
$$

and vice versa

$$
\gamma_{12}=\beta_{12345}-\beta_{21345}
$$

which implies that the $\beta_{i j k l m}$ satisfy

$$
\beta_{[i j][k l] m}=0 .
$$

In the next section, we will use these properties to prove a one-loop relation between subleadingcolor SYM and supergravity five-point amplitudes.

\section{One-loop supergravity-SYM relation}

Given the $\mathcal{N}=4$ SYM amplitude in a form (3.4) satisfying color-kinematic duality, the BCJ conjecture [8] holds that the $\mathcal{N}=8$ supergravity amplitude can be written as a double copy [18]

$$
\mathcal{M}_{5}^{(1)}=-\left(\frac{\kappa}{2}\right)^{5} \sum_{S_{5}}\left(\frac{1}{10} \tilde{\beta}_{12345} \beta_{12345} I^{(P)}(12345)+\frac{1}{4} \tilde{\gamma}_{12 ; 345} \gamma_{12 ; 345} I^{(B)}(12 ; 345)\right)
$$

where $\tilde{\beta}_{12345}$ and $\tilde{\gamma}_{12 ; 345}$ correspond to a second copy of the $\mathcal{N}=4$ SYM numerators. It was verified in refs. [18, 20] that eq. (4.1) is equivalent to the known five-point amplitude [4]. The additional symmetries of $\gamma_{i j}$ and $\tilde{\gamma}_{i j}$, allow one to rewrite eq. (4.1) as

$$
\mathcal{M}_{5}^{(1)}=-\left(\frac{\kappa}{2}\right)^{5}\left(\sum_{S_{5} / \mathbb{Z}_{5} \times \mathbb{Z}_{2}} \tilde{\beta}_{12345} \beta_{12345} I^{(P)}(12345)+\sum_{S_{5} / \mathbb{Z}_{2} \times S_{3}} \tilde{\gamma}_{12} \gamma_{12} I^{(B)}(12)\right)
$$

where $I^{(B)}(12)$ is defined in eq. (3.12).

The one-loop supergravity amplitude has a $1 / \epsilon$ IR divergence [25], and so it is plausible that it may be expressed as a linear combination of the double-trace (subleading-in- $1 / N$ ) color-ordered amplitudes $A_{5 ; 3}(12 ; 345)$, which also have a $1 / \epsilon$ leading IR divergence. We now establish such a relation. 
Consider the following linear combination of double-trace coefficients

$$
\begin{aligned}
\sum_{S_{5}} \tilde{\beta}_{12345} A_{5 ; 3}(12 ; 345) & =\sum_{S_{5}} \frac{1}{2}\left(\tilde{\beta}_{12345}-\tilde{\beta}_{12435}\right) A_{5 ; 3}(12 ; 345) \\
& =\sum_{S_{5}} \frac{1}{2}\left(\tilde{\beta}_{34512}-\tilde{\beta}_{43512}\right) A_{5 ; 3}(12 ; 345) \\
& =\sum_{S_{5}} \frac{1}{2} \tilde{\gamma}_{34} A_{5 ; 3}(12 ; 345) \\
& =\sum_{S_{5}} \frac{1}{6}\left(\tilde{\gamma}_{34}+\tilde{\gamma}_{45}+\tilde{\gamma}_{53}\right) A_{5 ; 3}(12 ; 345)
\end{aligned}
$$

where we have used eq. (3.15) as well as the symmetries of $A_{5 ; 3}(12 ; 345)$ and $\beta_{12345}$. Since $\tilde{\gamma}_{34}+\tilde{\gamma}_{45}+\tilde{\gamma}_{53}$ has the same symmetries as $A_{5 ; 3}(12 ; 345)$, we can restrict the sum to the ten independent double-trace coefficients

$$
\sum_{S_{5}} \tilde{\beta}_{12345} A_{5 ; 3}(12 ; 345)=\sum_{S_{5} / \mathbb{Z}_{2} \times S_{3}} 2\left(\tilde{\gamma}_{34}+\tilde{\gamma}_{45}+\tilde{\gamma}_{53}\right) A_{5 ; 3}(12 ; 345) .
$$

Each of the ten subleading-color amplitudes $A_{5: 3}(12 ; 345)$ appearing in eq. (4.4) can be rewritten in terms of pentagon integrals $I^{(P)}(i j k l m)$ and (sums of) box integrals $I^{(B)}(i j)$ using eq. (3.10).

First we extract the coefficient of $I^{(P)}(12345)$ in the double-trace amplitude $A_{5 ; 3}(\mathrm{ij} ; \mathrm{klm})$. These can be read off the first row of eq. (2.11). Each of the following subleading-color amplitudes contain a factor of $\beta_{12345} I^{(P)}(12345)$ :

$$
\begin{array}{lllll}
A_{5: 3}(12 ; 345), & A_{5: 3}(23 ; 451), & A_{5: 3}(34 ; 512), & A_{5: 3}(45 ; 123), & A_{5: 3}(51 ; 234), \\
A_{5: 3}(13 ; 245), & A_{5: 3}(24 ; 351), & A_{5: 3}(35 ; 412), & A_{5: 3}(41 ; 523), & A_{5: 3}(52 ; 134) .
\end{array}
$$

Hence the coefficient of $i g^{5} I^{(P)}(12345)$ in $\sum_{S_{5}} \tilde{\beta}_{12345} A_{5 ; 3}(12 ; 345)$ is

$$
\begin{aligned}
& 2\left[\left(\tilde{\gamma}_{34}+\tilde{\gamma}_{45}+\tilde{\gamma}_{53}\right)+\left(\tilde{\gamma}_{45}+\tilde{\gamma}_{51}+\tilde{\gamma}_{14}\right)+\left(\tilde{\gamma}_{51}+\tilde{\gamma}_{12}+\tilde{\gamma}_{25}\right)+\left(\tilde{\gamma}_{12}+\tilde{\gamma}_{23}+\tilde{\gamma}_{31}\right)\right. \\
& +\left(\tilde{\gamma}_{23}+\tilde{\gamma}_{34}+\tilde{\gamma}_{42}\right)+\left(\tilde{\gamma}_{24}+\tilde{\gamma}_{45}+\tilde{\gamma}_{52}\right)+\left(\tilde{\gamma}_{35}+\tilde{\gamma}_{51}+\tilde{\gamma}_{13}\right)+\left(\tilde{\gamma}_{41}+\tilde{\gamma}_{12}+\tilde{\gamma}_{24}\right) \\
& \left.+\left(\tilde{\gamma}_{52}+\tilde{\gamma}_{23}+\tilde{\gamma}_{35}\right)+\left(\tilde{\gamma}_{13}+\tilde{\gamma}_{34}+\tilde{\gamma}_{41}\right)\right] \beta_{12345} \\
= & 10\left(\tilde{\gamma}_{12}+\tilde{\gamma}_{13}+\tilde{\gamma}_{14}+\tilde{\gamma}_{23}+\tilde{\gamma}_{24}+\tilde{\gamma}_{34}\right) \beta_{12345} \\
= & 20 \tilde{\beta}_{12345} \beta_{12345}
\end{aligned}
$$

where we used eqs. (3.13) and (3.14) in the last two lines.

Next we extract the coefficient of $I^{(B)}(34)$ in eq. (4.4). The three independent subleading-color amplitudes that contribute to the coefficient of $I^{(B)}(34)$ are

$$
\begin{aligned}
& \left.A_{5 ; 3}(12 ; 345)\right|_{\text {box }}=2\left[\gamma_{34} I^{(B)}(34)+\gamma_{45} I^{(B)}(45)+\gamma_{53} I^{(B)}(53)\right] \\
& \left.A_{5 ; 3}(25 ; 341)\right|_{\text {box }}=2\left[\gamma_{34} I^{(B)}(34)+\gamma_{41} I^{(B)}(41)+\gamma_{13} I^{(B)}(13)\right] \\
& \left.A_{5 ; 3}(51 ; 342)\right|_{\text {box }}=2\left[\gamma_{34} I^{(B)}(34)+\gamma_{42} I^{(B)}(42)+\gamma_{23} I^{(B)}(23)\right] .
\end{aligned}
$$


Hence the coefficient of $i g^{5} I^{(B)}(34)$ in $\sum_{S_{5}} \tilde{\beta}_{12345} A_{5 ; 3}(12 ; 345)$ is

$$
\begin{aligned}
& 4\left[\left(\tilde{\gamma}_{34}+\tilde{\gamma}_{45}+\tilde{\gamma}_{53}\right)+\left(\tilde{\gamma}_{34}+\tilde{\gamma}_{41}+\tilde{\gamma}_{13}\right)+\left(\tilde{\gamma}_{34}+\tilde{\gamma}_{42}+\tilde{\gamma}_{23}\right)\right] \gamma_{34} \\
= & 12 \tilde{\gamma}_{34} \gamma_{34}+4\left(\tilde{\gamma}_{45}+\tilde{\gamma}_{41}+\tilde{\gamma}_{42}\right) \gamma_{34}+4\left(\tilde{\gamma}_{53}+\tilde{\gamma}_{13}+\tilde{\gamma}_{23}\right) \gamma_{34} \\
= & 20 \tilde{\gamma}_{34} \gamma_{34}
\end{aligned}
$$

where we used eq. (3.13) in the last line.

Assembling all the pieces we have

$$
\sum_{S_{5}} \tilde{\beta}_{12345} A_{5 ; 3}(12 ; 345)=20 i g^{5}\left(\sum_{S_{5} / \mathbb{Z}_{5} \times \mathbb{Z}_{2}} \tilde{\beta}_{12345} \beta_{12345} I^{(P)}(12345)+\sum_{S_{5} / \mathbb{Z}_{2} \times S_{3}} \tilde{\gamma}_{12} \gamma_{12} I^{(B)}(12)\right) .
$$

Comparing this with the one-loop supergravity amplitude (4.2), we obtain the SYM-supergravity relation

$$
\mathcal{M}_{5}^{(1)}=\frac{-1}{20 i g^{5}}\left(\frac{\kappa}{2}\right)^{5} \sum_{S_{5}} \tilde{\beta}_{12345} A_{5 ; 3}(12 ; 345)
$$

which is the five-point generalization of the one-loop SYM-supergravity relation for the four-point amplitude [2,22].

We note that eq. (4.10) is distinct from the relation between the one-loop supergravity amplitude and the leading-color SYM amplitudes

$$
\mathcal{M}_{5}^{(1)}=\frac{-1}{i g^{5}}\left(\frac{\kappa}{2}\right)^{5} \sum_{S_{5} / \mathbb{Z}_{5} \times \mathbb{Z}_{2}} \tilde{\beta}_{12345} A_{5 ; 1}(12345)
$$

proposed in eq. (3.27) of ref. [20]. Indeed, substituting eq. (3.9) into eq. (4.11) one finds

$$
\begin{aligned}
\mathcal{M}_{5}^{(1)}=-\left(\frac{\kappa}{2}\right)^{5} \sum_{S_{5}} & \frac{1}{10} \tilde{\beta}_{12345}\left(\beta_{12345} I^{(P)}(12345)+5 \gamma_{12 ; 345} I^{(B)}(12 ; 345)\right) \\
=-\left(\frac{\kappa}{2}\right)^{5} \sum_{S_{5}} & {\left[\frac{1}{10} \tilde{\beta}_{12345} \beta_{12345} I^{(P)}(12345)\right.} \\
+ & \left.\frac{1}{8}\left(\tilde{\beta}_{12345}-\tilde{\beta}_{21345}+\tilde{\beta}_{12543}-\tilde{\beta}_{21543}\right) \gamma_{12 ; 345} I^{(B)}(12 ; 345)\right] .
\end{aligned}
$$

Then by using the symmetries of $\tilde{\beta}_{12345}$ together with the relation (3.15) one verifies that this is precisely equal to eq. (4.1), thus providing a direct verification of the equivalence of eqs. (3.27) and (3.28) of ref. [20]. Because the leading-color SYM amplitudes goes as $1 / \epsilon^{2}$, the leading IR divergences must cancel between the terms of eq. (4.11), whereas in eq. (4.10) the individual terms all go as $1 / \epsilon$. 


\section{Conclusions}

In this paper, we have recast the Carrasco-Johansson representation of the one-loop $\mathcal{N}=4$ SYM five-point amplitude, which respects color-kinematic duality, into a trace basis consisting of leading- and subleading-color partial amplitudes. We then proposed and proved a linear relation between the one-loop $\mathcal{N}=8$ supergravity five-point amplitude and the subleading-color SYM amplitude, guided by the fact that the leading IR divergence of each goes as $1 / \epsilon$. This generalizes earlier one- and two-loop relations for four-point amplitudes [2, 22,. In all of these cases, the kinematic numerator factors are loop-momentum-independent and so we were able to obtain relations between integrated amplitudes, and not just integrands. It is a challenging problem to find similar relations when numerator factors depend on loop momenta. Focusing on the IR behavior of the supergravity and subleading-color SYM amplitudes may provide clues for discovering further supergravity-SYM relations.

\section{Acknowledgments}

We would like to thank H. Nastase for his collaboration on our previous work on SYM-supergravity relations.

\section{A IR divergence of the subleading-color amplitude}

In this appendix, we rederive the result [24] that the leading IR divergence of the one-loop subleading-color five-point amplitude $A_{5 ; 3}(12 ; 345)$ goes as $1 / \epsilon$. Recall from eq. (2.17) that the one-loop five-point coefficients satisfy

$$
A_{13}^{(1)}=A_{1}^{(1)}-A_{2}^{(1)}+A_{3}^{(1)}-A_{4}^{(1)}-A_{5}^{(1)}+A_{6}^{(1)}-A_{7}^{(1)}+A_{8}^{(1)}-A_{9}^{(1)}+A_{10}^{(1)}+A_{11}^{(1)}-A_{12}^{(1)} .
$$

Using this relation, it was shown in ref. [24] that the subleading-color amplitude $A_{5 ; 3}(12 ; 345)$ can be interpreted as sums of volumes of simple polytopes [28,29].

We know from ref. [21] that the one-loop planar five-point amplitude has a Laurent expansion beginning

$$
A_{i}^{(1)}=-\frac{5 g^{2} N}{32 \pi^{2} \epsilon^{2}} A_{i}^{(0)}+\mathcal{O}\left(\frac{1}{\epsilon}\right), \quad i=1, \cdots 12
$$

where $A_{i}^{(0)}$ is the tree-level amplitude, so

$$
\begin{aligned}
A_{13}^{(1)}=-\frac{5 g^{2} N}{32 \pi^{2} \epsilon^{2}} & \left(A_{1}^{(0)}-A_{2}^{(0)}+A_{3}^{(0)}-A_{4}^{(0)}-A_{5}^{(0)}+A_{6}^{(0)}\right. \\
& \left.-A_{7}^{(0)}+A_{8}^{(0)}-A_{9}^{(0)}+A_{10}^{(0)}+A_{11}^{(0)}-A_{12}^{(0)}\right)+\mathcal{O}\left(\frac{1}{\epsilon}\right) .
\end{aligned}
$$

The twelve tree-level coefficients appearing in this equation are not independent, however, but are related the Kleiss-Kuijf relations [30]. A nice way to derive these relations is to write the 
tree-level amplitude in a color basis consisting of [26,31]

$$
\begin{array}{llll}
c_{1}=\tilde{f}^{a_{1} a_{2} b} \tilde{f}^{b a_{3} c} \tilde{f}^{c a_{4} a_{5}}, & c_{2}=\tilde{f}^{a_{1} a_{4} b} \tilde{f}^{b a_{3} c} \tilde{f}^{c a_{2} a_{5}}, & c_{3}=\tilde{f}^{a_{1} a_{3} b} \tilde{f}^{b a_{4} c} \tilde{f}^{c a_{2} a_{5}}, \\
c_{4}=\tilde{f}^{a_{1} a_{2} b} \tilde{f}^{b a_{4} c} \tilde{f}^{c a_{3} a_{5}}, & c_{5}=\tilde{f}^{a_{1} a_{4} b} \tilde{f}^{b a_{2} c} \tilde{f}^{c a_{3} a_{5}}, & c_{6}=\tilde{f}^{a_{1} a_{3} b} \tilde{f}^{b a_{2} c} \tilde{f}^{c a_{4} a_{5}} .
\end{array}
$$

(These six basis elements are denoted $\left\{c_{1}, c_{6}, c_{9}, c_{12}, c_{14}, c_{15}\right\}$ in ref. [7]. The other nine $c_{i}$ are related to these by Jacobi identities.) This color basis can be rewritten using a basis of singletrace terms

$$
c_{i}=\sum_{\lambda=1}^{12} M_{i \lambda} t_{\lambda}
$$

where $t_{\lambda}$ are defined as in eq. (2.7) except that the factor of $N$ is omitted at tree level. Then

$$
M_{i \lambda}=\left(\begin{array}{llllllllllll}
1 & 0 & 0 & 0 & 0 & 0 & 1 & 0 & 0 & 0 & 1 & 1 \\
0 & 1 & 0 & 0 & 0 & 0 & 0 & 1 & 1 & 1 & 0 & 0 \\
0 & 0 & 1 & 0 & 0 & 0 & 1 & 1 & 1 & 0 & 0 & 0 \\
0 & 0 & 0 & 1 & 0 & 0 & 0 & 0 & 0 & 1 & 1 & 1 \\
0 & 0 & 0 & 0 & 1 & 0 & 0 & 0 & 1 & 1 & 1 & 0 \\
0 & 0 & 0 & 0 & 0 & 1 & 1 & 1 & 0 & 0 & 0 & 1
\end{array}\right) .
$$

This rank-six matrix possesses six null eigenvectors $R_{\lambda j}$ :

$$
\sum_{\lambda=1}^{12} M_{i \lambda} R_{\lambda j}=0, \quad j=1, \cdots 6 .
$$

Given that $M_{i \lambda}$ has the form $\left(\begin{array}{ll}\mathbb{1}_{6 \times 6} & m\end{array}\right)$, one sees that $R=\left(\begin{array}{c}-m \\ \mathbb{1}_{6 \times 6}\end{array}\right)$. The existence of these null eigenvectors, together with eq. (2.12), implies six relations among the color-ordered amplitudes

$$
\sum_{\lambda=1}^{12} A_{\lambda}^{(0)} R_{\lambda j}=0, \quad j=1, \cdots 6
$$

which are precisely the Kleiss-Kuijf relations [30 at five points. These relations can be used to eliminate $A_{7}^{(0)}$ through $A_{12}^{(0)}$, e.g., $A_{7}^{(0)}=A_{1}^{(0)}+A_{3}^{(0)}+A_{6}^{(0)}$. Substituting these relations into eq. (A.3) demonstrates that the leading $1 / \epsilon^{2}$ IR divergence of $A_{5 ; 3}(12 ; 345)$ vanishes. 


\section{References}

[1] H. Kawai, D. C. Lewellen and S. H. H. Tye, "A Relation Between Tree Amplitudes of Closed and Open Strings", Nucl. Phys. B269, 1 (1986).

[2] M. B. Green, J. H. Schwarz and L. Brink, "N=4 Yang-Mills and N=8 Supergravity as Limits of String Theories", Nucl. Phys. B198, 474 (1982).

[3] Z. Bern, L. J. Dixon, D. C. Dunbar, M. Perelstein and J. S. Rozowsky, "On the relationship between Yang-Mills theory and gravity and its implication for ultraviolet divergences", Nucl. Phys. B530, 401 (1998), hep-th/9802162.

[4] Z. Bern, L. J. Dixon, M. Perelstein and J. S. Rozowsky, "Multi-leg one-loop gravity amplitudes from gauge theory", Nucl. Phys. B546, 423 (1999), hep-th/9811140.

[5] Z. Bern, J. J. M. Carrasco, L. J. Dixon, H. Johansson and R. Roiban, "Manifest Ultraviolet Behavior for the Three-Loop Four-Point Amplitude of N=8 Supergravity", Phys. Rev. D78, 105019 (2008), arxiv:0808.4112.

[6] Z. Bern, J. J. M. Carrasco, L. J. Dixon, H. Johansson and R. Roiban, "The Complete Four-Loop Four-Point Amplitude in N=4 Super- Yang-Mills Theory", Phys. Rev. D82, 125040 (2010), arxiv:1008.3327.

[7] Z. Bern, J. J. M. Carrasco and H. Johansson, "New Relations for Gauge-Theory Amplitudes", Phys. Rev. D78, 085011 (2008), arxiv:0805.3993.

[8] Z. Bern, J. J. M. Carrasco and H. Johansson, "Perturbative Quantum Gravity as a Double Copy of Gauge Theory", Phys. Rev. Lett. 105, 061602 (2010), arxiv:1004.0476.

[9] N. E. J. Bjerrum-Bohr, P. H. Damgaard and P. Vanhove, "Minimal Basis for Gauge Theory Amplitudes", Phys. Rev. Lett. 103, 161602 (2009), arxiv:0907.1425.

[10] S. Stieberger, "Open and Closed vs. Pure Open String Disk Amplitudes", arxiv:0907.2211.

[11] B. Feng, R. Huang and Y. Jia, "Gauge Amplitude Identities by On-shell Recursion Relation in S-matrix Program", Phys. Lett. B695, 350 (2011), arxiv:1004.3417.

[12] Y.-X. Chen, Y.-J. Du and B. Feng, "A Proof of the Explicit Minimal-basis Expansion of Tree Amplitudes in Gauge Field Theory", JHEP 1102, 112 (2011), arxiv:1101.0009.

[13] Z. Bern, T. Dennen, Y.-t. Huang and M. Kiermaier, "Gravity as the Square of Gauge Theory", Phys. Rev. D82, 065003 (2010), arxiv: 1004.0693.

[14] J. J. M. Carrasco and H. Johansson, "Generic multiloop methods and application to $N=4$ superYang-Mills", arxiv:1103.3298.

[15] T. Sondergaard, "New Relations for Gauge-Theory Amplitudes with Matter", Nucl. Phys. B821, 417 (2009), arxiv:0903.5453;

C. R. Mafra, "Simplifying the Tree-level Superstring Massless Five-point Amplitude", JHEP 1001, 007 (2010), arxiv:0909.5206,

S. H. Henry Tye and Y. Zhang, "Dual Identities inside the Gluon and the Graviton Scattering Amplitudes", JHEP 1006, 071 (2010), arxiv:1003.1732;

N. E. J. Bjerrum-Bohr, P. H. Damgaard, T. Sondergaard and P. Vanhove, "Monodromy and Jacobi-like Relations for Color-Ordered Amplitudes", JHEP 1006, 003 (2010), arxiv:1003.2403, 
P. Vanhove, "The critical ultraviolet behaviour of $N=8$ supergravity amplitudes",

arxiv:1004.1392,

Y. Jia, R. Huang and C.-Y. Liu, "U(1)-decoupling, $K K$ and BCJ relations in $\mathcal{N}=4 S Y M "$,

Phys. Rev. D82, 065001 (2010), arxiv:1005.1821;

N. E. J. Bjerrum-Bohr, P. H. Damgaard, B. Feng and T. Sondergaard, "Gravity and Yang-Mills Amplitude Relations", Phys. Rev. D82, 107702 (2010), arxiv:1005.4367;

N. E. J. Bjerrum-Bohr, P. H. Damgaard, B. Feng and T. Sondergaard, "New Identities among

Gauge Theory Amplitudes", Phys. Lett. B691, 268 (2010), arxiv:1006.3214;

B. Feng and S. He, "KLT and New Relations for N=8 SUGRA and N=4 SYM",

JHEP 1009, 043 (2010), arxiv: 1007.0055,

H. Tye and Y. Zhang, "Comment on the Identities of the Gluon Tree Amplitudes",

arxiv:1007.0597,

N. E. J. Bjerrum-Bohr, P. H. Damgaard, B. Feng and T. Sondergaard, "Proof of Gravity and

Yang-Mills Amplitude Relations", JHEP 1009, 067 (2010), arxiv:1007.3111;

D. Vaman and Y.-P. Yao, "Constraints and Generalized Gauge Transformations on Tree- Level

Gluon and Graviton Amplitudes", JHEP 1011, 028 (2010), arxiv:1007.3475;

C. R. Mafra, "Towards Field Theory Amplitudes From the Cohomology of Pure Spinor

Superspace", JHEP 1011, 096 (2010), arxiv:1007.3639;

C. R. Mafra, "PSS: A FORM Program to Evaluate Pure Spinor Superspace Expressions",

arxiv:1007.4999;

B. Feng, S. He, R. Huang and Y. Jia, "Note on New KLT relations", JHEP 1010, 109 (2010),

arxiv:1008.1626:

Y. Abe, "Holonomies of gauge fields in twistor space 3: gravity as a square of $N=4$ theory",

Nucl. Phys. B842, 475 (2011), arxiv: 1008.2800;

J.-H. Huang, R. Huang and Y. Jia, "Tree amplitudes of noncommutative $U(N)$ Yang-Mills

Theory", arxiv:1009.5073;

J. Bjornsson, "Multi-loop amplitudes in maximally supersymmetric pure spinor field theory", JHEP 1101, 002 (2011), arxiv:1009.5906:

N. E. J. Bjerrum-Bohr, P. H. Damgaard, T. Sondergaard and P. Vanhove, "The Momentum

Kernel of Gauge and Gravity Theories", JHEP 1101, 001 (2011), arxiv:1010.3933;

C. R. Mafra, O. Schlotterer, S. Stieberger and D. Tsimpis, "Six Open String Disk Amplitude in

Pure Spinor Superspace”, Nucl. Phys. B846, 359 (2011), arxiv:1011.0994;

Y.-X. Chen, Y.-J. Du and B. Feng, "On tree amplitudes with gluons coupled to gravitons",

JHEP 1101, 081 (2011), arxiv:1011.1953;

H. Nastase and H. J. Schnitzer, "On KLT and SYM-supergravity relations from 5-point 1-loop amplitudes", JHEP 1101, 048 (2011), arxiv:1011.2487;

N. E. J. Bjerrum-Bohr, P. H. Damgaard, B. Feng and T. Sondergaard, "Unusual identities for QCD at tree-level", J. Phys. Conf. Ser. 287, 012030 (2011), arxiv:1101.5555,

Z. Bern and T. Dennen, "A Color Dual Form for Gauge-Theory Amplitudes", arxiv:1103.0312;

J. Broedel and R. Kallosh, "From lightcone actions to maximally supersymmetric amplitudes", JHEP 1106, 024 (2011), arxiv:1103.0322,

N. E. J. Bjerrum-Bohr, P. H. Damgaard, H. Johansson and T. Sondergaard, "Monodromy-like

Relations for Finite Loop Amplitudes", JHEP 1105, 039 (2011), arxiv:1103.6190;

C. R. Mafra, O. Schlotterer and S. Stieberger, "Explicit BCJ Numerators from Pure Spinors", JHEP 1107, 092 (2011), arxiv: 1104.5224; 
R. Monteiro and D. O'Connell, "The Kinematic Algebra From the Self-Dual Sector", JHEP 1107, 007 (2011), arxiv:1105.2565;

Y.-J. Du, B. Feng and C.-H. Fu, "BCJ Relation of Color Scalar Theory and KLT Relation of Gauge Theory", arxiv:1105.3503,

C. R. Mafra, O. Schlotterer and S. Stieberger, "Complete N-Point Superstring Disk Amplitude I. Pure Spinor Computation", arxiv:1106.2645

[16] J. Broedel and J. J. M. Carrasco, "Virtuous Trees at Five and Six Points for Yang-Mills and Gravity", arxiv:1107.4802.

[17] T. Sondergaard, "Perturbative Gravity and Gauge Theory Relations - A Review", arxiv:1106.0033.

[18] J. J. M. Carrasco and H. Johansson, "Five-Point Amplitudes in N=4 Super-Yang-Mills Theory and $N=8$ Supergravity", arxiv:1106.4711.

[19] J. J. M. Carrasco and H. Johansson, to appear.

[20] Z. Bern, C. Boucher-Veronneau and H. Johansson, "N $\geq 4$ Supergravity Amplitudes from Gauge Theory at One Loop", arxiv:1107.1935.

[21] Z. Bern, L. J. Dixon, D. C. Dunbar and D. A. Kosower, "One-Loop n-Point Gauge Theory Amplitudes, Unitarity and Collinear Limits", Nucl. Phys. B425, 217 (1994), hep-ph/9403226.

[22] S. G. Naculich, H. Nastase and H. J. Schnitzer, "Subleading-color contributions to gluon-gluon scattering in $N=4 S Y M$ theory and relations to $N=8$ supergravity", JHEP 0811, 018 (2008), arxiv:0809.0376.

[23] S. G. Naculich and H. J. Schnitzer, "IR divergences and Regge limits of subleading-color contributions to the four-gluon amplitude in N=4 SYM Theory", JHEP 0910, 048 (2009) arxiv:0907.1895,

[24] H. Nastase and H. J. Schnitzer, "Twistor and Polytope Interpretations for Subleading Color One-Loop Amplitudes", arxiv:1104.2752.

[25] D. C. Dunbar and P. S. Norridge, "Infinities within graviton scattering amplitudes", Class. Quant. Grav. 14, 351 (1997), hep-th/9512084.

[26] V. Del Duca, L. J. Dixon and F. Maltoni, "New color decompositions for gauge amplitudes at tree and loop level", Nucl. Phys. B571, 51 (2000), hep-ph/9910563.

[27] Z. Bern and D. A. Kosower, "Color decomposition of one loop amplitudes in gauge theories", Nucl.Phys. B362, 389 (1991).

[28] N. Arkani-Hamed, J. L. Bourjaily, F. Cachazo, A. Hodges and J. Trnka, "A Note on Polytopes for Scattering Amplitudes", arxiv:1012.6030.

[29] L. Mason and D. Skinner, "Amplitudes at Weak Coupling as Polytopes in $A_{d} S_{5}$ ", J. Phys. A44, 135401 (2011), arxiv:1004.3498.

[30] R. Kleiss and H. Kuijf, "Multi-gluon cross-sections and five jet production at hadron colliders", Nucl. Phys. B312, 616 (1989).

[31] V. Del Duca, A. Frizzo and F. Maltoni, "Factorization of tree QCD amplitudes in the high-energy limit and in the collinear limit", Nucl. Phys. B568, 211 (2000), hep-ph/9909464. 